\title{
Axially symmetric elasticity problems for the hollow cylinder with the stress-free ends. Analytical solving via a variational method of homogeneous solutions
}

\author{
Chekurin V. F. ${ }^{1,2}$, Postolaki L. I. ${ }^{1}$ \\ ${ }^{1}$ Pidstryhach Institute for Applied Problems of Mechanics and Mathematics, \\ National Academy of Sciences of Ukraine, \\ 3-b Naukova Str., 79060, Lviv, Ukraine \\ ${ }^{2}$ Kujawy and Pomorze University in Bydgoszcz, \\ 55-57 Torunska Str., 85-023, Bydgoszcz, Poland
}

(Received 11 June 2019; Revised 20 January 2020; Accepted 21 January 2020)

\begin{abstract}
An axially symmetric problem for a hollow cylinder with unloaded bases is considered. On the inner and outer cylindrical surfaces, the normal and tangential loads are prescribed. The problem is reduced to a biharmonic equation with corresponding boundary conditions. Application of the method of variables separation results in a homogeneous boundary value problem for the ordinary differential equation. Its eigenfunctions have been used to construct an infinite system of homogeneous solutions for the initial biharmonic problem. Its solution, represented as a series expansion in terms of homogeneous solutions, depends on four infinite sequences of real constants. To determine them, the variational method has been applied, in which the subordination of the solution to the boundary conditions, given on cylindrical surfaces, is performed in the norm $L_{2}$. It brings to an infinite system of algebraic equations which has been solved by the reduction method. The quantitative studies have confirmed the good convergence of the method.
\end{abstract}

Keywords: elasticity problem, hollow cylinder, variational method of homogeneous solutions.

2010 MSC: $35 J 30$

DOI: $10.23939 / \mathrm{mmc} 2020.01 .048$

\section{Introduction}

Despite the intensive development of software systems that implement various numerical methods, analytical solutions of boundary value problems still remain used in solid mechanics. There are several reasons for this: precise analytical solutions are indispensable tools for testing numerical methods; it is easier on their basis to obtain approximate formulae for engineering applications; many nonlinear problems can be reduced to sequences of linear ones that can be effectively solved analytically. It is also important that the development of new analytical methods shapes the scientific basis for the creation of new software systems for the quantitative analysis of new classes of direct and inverse problems, formulated in the framework of new mathematical models that are not provided in the existing software environments.

By analyzing the known analytical approaches to the study of the axially symmetric strain-stressed state of the hollow cylinder, several classes of problems can be identified.

The first is the linear plane axially symmetric problems. Their solutions are functions that depend only on the radial coordinate. Among them, in particular, is the problem [1] formulated within the framework of an elasticity model, which takes into account both macro- and micro-stresses in a media

This work was supported by the grant on the National Academy of Sciences of Ukraine "Mathematical models, variational and iteration methods for solving of direct and inverse problems for theory of field interaction in solid bodies" (Number of State Registration 0119U100675). 
with microstructure. To this class, we also attribute the axially symmetric elasticity problems for the thick-walled cylinder with radial-dependent material characteristics (see papers $[2-4]$ and the reviews of recent publications in this direction, presented in them).

The second class is linear axially symmetric problems for an infinite hollow cylinder under local loading [5,6]. Analytical solutions for them are being obtained using the Fourier transform. In this connection, we also note the publication [7], in which the contact axially symmetric problem for composite axially-homogeneous infinite cylinder, resting under a local loading applying to its outer surface, is considered.

To the third class, we attribute axially symmetric problems for a finite hollow cylinder. In the paper [8], an exact solution of such a problem was obtained using the method of cross-superposition. By this method, the components of displacement vector are represented as expansions in complete systems of orthogonal functions (trigonometric, hyperbolic and cylindrical). It allowed us to precisely satisfy the boundary conditions both on the cylindrical surfaces and at the ends of the cylinder. In [9] an axially symmetric problem for the finite hollow cylinder with traction-free ends is considered. It was solved by applying the representation of the solution in the form of expansions in systems of homogeneous solutions.

Homogeneous solutions are systems of functions that satisfy the equation of the theory of elasticity in body volume and homogeneous conditions on a part of the body surface. They came to such function considering two-dimensional problems for a rectangular domain, on two parallel sides of which homogeneous conditions in stresses or displacements are prescribed (see literature reviews in the monograph [10] and article [11]). Using some special representations of the solution in form of separated variables and subordinating them to the homogeneous conditions given at two opposite sides, one can come to complete systems of eigenfunctions that satisfy some integral conditions (quasi-orthogonality relations) [10]. This allows a series expansion of the problem solution in terms of the eigenfunctions. Such representation of the solution automatically satisfies all problem equations within the domain as well as the homogeneous conditions prescribed at two sides of its boundary. The expansion coefficients can be determined by subordinating the solution to the non-homogeneous boundary conditions prescribed at other two sides of the boundary. But, applying the quasi-orthogonality conditions makes it possible obtaining closed-form formulae for the expansion coefficients only for the mixed boundary conditions (when normal displacement and tangential traction, or, vice versa, normal traction and tangential displacement are prescribed at two opposite sides of the rectangle).

In the variational method of homogeneous solutions [12,13], the solution of the problem, presented in the form of the series expansion in terms of eigenfunctions, is subordinated to non-uniform boundary conditions without using the point-wise convergence, but applying the convergence in the quadratic norm. With this aim, a functional is introduced. It defines in norm $L_{2}$ the deviation of the problem solution from all specified boundary conditions. The minimum conditions of the functional lead to an infinite system of linear algebraic equations for the expansion coefficients. The system can be solved using the methods developed in monograph [14]. The variation method is applicable to the boundary conditions in stresses, displacements and mixed ones. Solutions for some contact $[15,16]$ and inverse $[12,17-19]$ problems were obtained with its application.

In [20-22], the variational method of homogeneous solutions was extended to axially symmetric elasticity problems for the solid cylinder of semi-infinite and finite length, with the traction-free lateral boundary and loaded end faces. In [23], this method was used to solve an inverse problem for determining axially symmetric residual stresses in a finite solid cylinder with the use of the data obtained by the photo-elasticity method.

In this paper, we develop the variational method of homogeneous solutions for a finite hollow cylinder, on the ends of which the homogeneous conditions in stresses are prescribed. The inner and outer cylindrical surfaces of the body are subjected to normal and tangential tractions. We reduced the problems to biharmonic ones. 


\section{Problem formulation}

We consider axially symmetric elasticity problem for the hollow cylinder of finite length $2 b \mathbb{V}=$ $\left\{r_{\text {in }} \leqslant r \leqslant r_{\text {out }}, 0 \leqslant \theta \leqslant 2 \pi,-b \leqslant z \leqslant b\right\}$, where $r, z$ and $\theta$ are radial, axial and hoop cylindrical coordinates, $r_{\text {out }}$ and $r_{\text {in }}$ stand for outer and inner radii of the cylinder. Let on the inner $r=r_{\text {in }}$ and outer $r=r_{\text {out }}$ cylindrical surfaces of the body the normal $\sigma_{\text {in }}(z)$ and $\sigma_{\text {out }}(z)$, tangential $\tau_{\text {in }}(z)$ and $\tau_{\text {out }}(z)$ tractions are prescribed:

$$
\left.\sigma_{r r}\right|_{r=r_{\text {in }}}=\sigma_{\text {in }}(z),\left.\quad \sigma_{r z}\right|_{r=r_{\text {in }}}=\tau_{\text {in }}(z),\left.\quad \sigma_{r r}\right|_{r=r_{\text {out }}}=\sigma_{\text {out }}(z),\left.\quad \sigma_{r z}\right|_{r=r_{\text {out }}}=\tau_{\text {out }}(z) .
$$

The end surfaces $z= \pm b$ of the cylinder are stress-free:

$$
\left.\sigma_{z z}\right|_{z= \pm b}=0,\left.\quad \sigma_{r z}\right|_{z= \pm b}=0 .
$$

Here $\sigma_{r r}, \sigma_{z z}, \sigma_{\theta \theta}, \sigma_{r z}$ stand for components of the stress tensor in cylindrical coordinates. They satisfy in domain $\mathbb{V}$ the equilibrium equations:

$$
\begin{aligned}
& \frac{1}{r} \frac{\partial}{\partial r}\left(r \sigma_{r r}\right)+\frac{\partial}{\partial z} \sigma_{r z}-\frac{1}{r} \sigma_{\theta \theta}=0, \\
& \frac{1}{r} \frac{\partial}{\partial r}\left(r \sigma_{r z}\right)+\frac{\partial}{\partial z} \sigma_{z z}=0
\end{aligned}
$$

and can be express via components of strain tensor $\varepsilon_{i j}$ by the elasticity relations

$$
\begin{aligned}
\sigma_{r r} & =\frac{E}{(1+\nu)(1-2 \nu)}\left((1-\nu) \varepsilon_{r r}+\nu\left(\varepsilon_{z z}+\varepsilon_{\theta \theta}\right)\right), \\
\sigma_{z z} & =\frac{E}{(1+\nu)(1-2 \nu)}\left((1-\nu) \varepsilon_{z z}+\nu\left(\varepsilon_{r r}+\varepsilon_{\theta \theta}\right)\right), \\
\sigma_{\theta \theta} & =\frac{E}{(1+\nu)(1-2 \nu)}\left((1-\nu) \varepsilon_{\theta \theta}+\nu\left(\varepsilon_{z z}+\varepsilon_{r r}\right)\right), \\
\sigma_{r z} & =\frac{E}{1+\nu} \varepsilon_{r z} .
\end{aligned}
$$

Here $E$ is Young's modulus, $\nu$ is Poisson's ratio.

Applying the Cauchy relations [24]

$$
\varepsilon_{r r}=\frac{\partial u_{r}}{\partial r}, \quad \varepsilon_{z z}=\frac{\partial u_{z}}{\partial z}, \quad \varepsilon_{\theta \theta}=\frac{u_{r}}{r}, \quad \varepsilon_{r z}=\frac{1}{2}\left(\frac{\partial u_{r}}{\partial z}+\frac{\partial u_{z}}{\partial r}\right) .
$$

we obtain from (3), (4) the governing equations in displacement for axially symmetric strain-stressed state [24]

$$
\begin{aligned}
& \nabla^{2} u_{r}+\frac{1}{1-2 \nu} \frac{\partial \varepsilon}{\partial r}-\frac{u_{r}}{r^{2}}=0 \\
& \nabla^{2} u_{z}+\frac{1}{1-2 \nu} \frac{\partial \varepsilon}{\partial z}=0
\end{aligned}
$$

Here $\varepsilon \equiv \varepsilon_{r r}+\varepsilon_{z z}+\varepsilon_{\theta \theta}=\frac{1}{r} \frac{\partial}{\partial r}\left(r u_{r}\right)-\frac{\partial u_{z}}{\partial z}, \nabla^{2} \equiv \frac{\partial^{2}}{\partial r^{2}}+\frac{1}{r} \frac{\partial}{\partial r}+\frac{\partial^{2}}{\partial z^{2}}$ is axially symmetric Laplace's operator.

To reduce the problem to non-dimensional form, we introduce the non-dimensional spatial coordinates $\xi \equiv r / r_{\text {out }}, \zeta \equiv z / r_{\text {out }}$, non-dimensional displacement $u \equiv u_{r} / r_{\text {out }}, v \equiv u_{z} / r_{\text {out }}$ and stress $\sigma \equiv \sigma_{r r} / E, \tau \equiv \sigma_{r z} / E$ components. We also introduce the other two non-dimensional components of stress tensor by the mappings $\sigma_{\theta \theta} / E \rightarrow \sigma_{\theta \theta}, \sigma_{z z} / E \rightarrow \sigma_{z z}$. 
Now to obtain relations (5) in non-dimensional form one should to put in them $E=1, \sigma_{r r}=\sigma$, $\sigma_{r z}=\tau$. Similarly, one should make the substitutions $u_{r}=u, u_{z}=v, \partial / \partial r \rightarrow \partial / \partial \xi$ and $\partial / \partial z \rightarrow \partial / \partial \zeta$ in relations (6) to reduce them to non-dimensional form.

It is convenient to use the Love function $\chi$ for the axially symmetric problem. Its dimension is $[N \cdot m]$. We introduce a non-dimensional Love function by the mapping $\chi \leftarrow \chi /\left(E r_{\text {out }}\right)$. With its using we can reduce the system (6) to the biharmonic equation

$$
\nabla^{2} \nabla^{2} \chi=0
$$

Non-dimensional components of the stress tensor and displacement vector are expressed via introduced function $\chi$ by (8) and (9) correspondingly

$$
\begin{aligned}
\sigma & =\frac{\partial}{\partial \zeta}\left(\nu \nabla^{2} \chi-\frac{\partial^{2} \chi}{\partial \xi^{2}}\right), & \tau & =\frac{\partial}{\partial \xi}\left((1-\nu) \nabla^{2} \chi-\frac{\partial^{2} \chi}{\partial \zeta^{2}}\right), \\
\sigma_{z z} & =\frac{\partial}{\partial \zeta}\left((2-\nu) \nabla^{2} \chi-\frac{\partial^{2} \chi}{\partial \zeta^{2}}\right), & \sigma_{\theta \theta} & =\frac{\partial}{\partial \zeta}\left(\nu \nabla^{2} \chi-\frac{1}{\xi} \frac{\partial \chi}{\partial \xi}\right) . \\
u & =-(1+\nu) \frac{\partial^{2} \chi}{\partial \xi \partial \zeta}, & v & =2\left(1-\nu^{2}\right) \nabla^{2} \chi-(1+\nu) \frac{\partial^{2} \chi}{\partial \zeta^{2}} .
\end{aligned}
$$

Using these formulas we obtain the presentation of the boundary conditions (1) and (2) in terms of the Love function (relations (10) and (11) correspondingly):

$$
\begin{aligned}
& \left.\frac{\partial}{\partial \zeta}\left(\nu \nabla^{2} \chi-\frac{\partial^{2} \chi}{\partial \xi^{2}}\right)\right|_{\xi=\xi_{\text {in }}}=\sigma_{\text {in }}(\zeta), \\
& \left.\frac{\partial}{\partial \xi}\left((1-\nu) \nabla^{2} \chi-\frac{\partial^{2} \chi}{\partial \zeta^{2}}\right)\right|_{\xi=\xi_{\text {in }}}=\tau_{\text {in }}(\zeta), \\
& \left.\frac{\partial}{\partial \zeta}\left(\nu \nabla^{2} \chi-\frac{\partial^{2} \chi}{\partial \xi^{2}}\right)\right|_{\xi=1}=\sigma_{\text {out }}(\zeta), \\
& \left.\frac{\partial}{\partial \xi}\left((1-\nu) \nabla^{2} \chi-\frac{\partial^{2} \chi}{\partial \zeta^{2}}\right)\right|_{\xi=1}=\tau_{\text {out }}(\zeta) \\
& \left.\frac{\partial}{\partial \zeta}\left((2-\nu) \nabla^{2} \chi-\frac{\partial^{2} \chi}{\partial \zeta^{2}}\right)\right|_{\zeta= \pm b}=0 \\
& \left.\frac{\partial}{\partial \xi}\left((1-\nu) \nabla^{2} \chi-\frac{\partial^{2} \chi}{\partial \zeta^{2}}\right)\right|_{\zeta= \pm b}=0
\end{aligned}
$$

Since the ends of the cylinder are unloaded, the tangential tractions on its lateral surface should satisfy the relation

$$
\int_{-b}^{b} \tau_{\text {in }}(\zeta) d \zeta+\int_{-b}^{b} \tau_{\text {out }}(\zeta) d \zeta=0
$$

To make the problem well-behaved it also is necessary to subordinate the functions of tangential tractions to the conditions

$$
\tau_{\text {in }}( \pm b)=0, \quad \tau_{\text {out }}( \pm b)=0
$$




\section{Eigenfunctions of axially symmetric biharmonic problems for a hollow cylinder}

\subsection{Separation of the variables}

We will seek the solution of biharmonic equation (7) in the form of separated variables [9]:

$$
\chi(\xi, \zeta)=h_{0}(\xi) \varphi(\zeta)
$$

choosing the radial function $h_{0}(\xi)$ in the form

$$
h_{0}(\xi)=C^{(1)} H_{0}^{(1)}(\gamma \xi)+C^{(2)} H_{0}^{(2)}(\gamma \xi),
$$

where $\varphi(\zeta)$ is unknown function, $C^{(1)}$ and $C^{(2)}$ are undefined constants, $H_{0}^{(1)}(\gamma \xi)$ and $H_{0}^{(2)}(\gamma \xi)$ stand for Hankel functions (Bessel functions of the third kind) [25], $\gamma$ is undefined constant.

After substitution of presentation (12) into relations (8), (9), we obtain

$$
\begin{aligned}
\sigma & =h_{0}(\xi)\left(\nu \varphi^{\prime \prime \prime}(\zeta)+(1-\nu) \gamma^{2} \varphi^{\prime}(\zeta)\right)-\frac{\gamma}{\xi} h_{1}(\xi) \varphi^{\prime}(\zeta), \\
\tau & =\gamma h_{1}(\xi)\left(\nu \varphi^{\prime \prime}(\zeta)+\gamma^{2}(1-\nu) \varphi(\zeta)\right), \\
\sigma_{z z} & =h_{0}(\xi)\left((1-\nu) \varphi^{\prime \prime \prime}(\zeta)-(2-\nu) \gamma^{2} \varphi^{\prime}(\zeta)\right), \\
\sigma_{\theta \theta} & =\nu h_{0}(\xi)\left(\varphi^{\prime \prime \prime}(\zeta)-\gamma^{2} \varphi^{\prime}(\zeta)\right)+\frac{\gamma}{\xi} h_{1}(\xi) \varphi^{\prime}(\zeta), \\
u & =(1+\nu) \gamma h_{1}(\xi) \varphi^{\prime}(\zeta), \\
v & =(1+\nu) h_{0}(\xi)\left((1-2 \nu) \varphi^{\prime \prime}(\zeta)+2 \gamma^{2}(\nu-1) \varphi(\zeta)\right),
\end{aligned}
$$

where $h_{1}(\xi)=C^{(1)} H_{1}^{(1)}(\gamma \xi)+C^{(2)} H_{1}^{(2)}(\gamma \xi)$.

Substituting (12) into equation (7), we come to the ordinary differential equation for axial function $\varphi(\zeta)$ :

$$
\varphi^{I V}(\zeta)-2 \gamma^{2} \varphi^{\prime \prime}(\zeta)+\gamma^{4} \varphi(\zeta)=0 .
$$

This equation also appears, when the method of homogeneous solutions is used to solve plane elasticity problems for a rectangular area, on two opposite sides of which homogeneous boundary conditions in stresses or displacement are prescribed $[10,12,13]$. Its general solution is the function

$$
\varphi(\zeta)=(A+B \zeta) \cosh (\gamma \zeta)+(C+D \zeta) \sinh (\gamma \zeta)
$$

dependent on four undefined constants $A, B, C, D$.

It is convenient to consider function $\varphi(\zeta)$ as the sum of its odd

$$
\varphi(\zeta)=L_{1} \sinh (\gamma \zeta)+L_{2} \zeta \cosh (\gamma \zeta)
$$

and even

$$
\varphi(\zeta)=L_{1} \cosh (\gamma \zeta)+L_{2} \zeta \sinh (\gamma \zeta)
$$

parts, both of which depend only on two undefined constants.

If function $\varphi(\zeta)$ in presentation (12) is taken in form (21), then function $\chi(\xi, \zeta)$ defines symmetrical with respect to the plane $\zeta=0$ strain-stressed state. In the other case, when $\varphi(\zeta)$ is taken in form $(22)$, $\chi(\xi, \zeta)$ determine the antisymmetric state of the cylinder.

Decomposing both the normal and tangential tractions of boundary conditions (10) on odd and even parts:

$$
\begin{aligned}
\sigma_{\text {in,out }}^{\text {odd }}(\zeta) & =1 / 2\left(\sigma_{\text {in out }}(\zeta)-\sigma_{\text {in,out }}(-\zeta)\right), & & \sigma_{\text {in,out }}^{\text {even }}(\zeta)=1 / 2\left(\sigma_{\text {in }, \text { out }}(\zeta)+\sigma_{\text {in,out }}(-\zeta)\right), \\
\tau_{\text {in,out }}^{\text {odd }}(\zeta) & =1 / 2\left(\tau_{\text {in,out }}(\zeta)-\tau_{\text {in,out }}(-\zeta)\right), & & \tau_{\text {in,out }}^{\text {even }}(\zeta)=1 / 2\left(\tau_{\text {in,out }}(\zeta)+\tau_{\text {in,out }}(-\zeta)\right)
\end{aligned}
$$


and applying the superposition method, we can split each of the initial biharmonic problem (10), (11) on the symmetrical and antisymmetric ones. In the symmetrical problems functions $\sigma_{\text {in,out }}^{\text {odd }}(\zeta)$ and $\tau_{\text {in,out }}^{\text {even }}(\zeta)$ should be used as boundary data in $(10)$, whereas in antisymmetric ones $-\sigma_{\text {ineout }}^{\text {even }}(\zeta)$ and $\tau_{\text {in,out }}^{\text {odd }}(\zeta)$. To determine the unknown constants in (21) and (22), we can utilize the boundary condition (11), prescribed on the end faces of the cylinder.

\subsection{The transcendental equations}

Taking the odd axial function $\varphi(\zeta)$ in presentation (12) and substituting obtained Love function $\chi(\xi, \zeta)$ into boundary conditions (11), we come to the homogeneous linear system of equations for the unknown coefficients in function (21):

$$
\left\{\begin{array}{l}
\gamma \cosh (\gamma b) L_{1}+((2 \nu-1) \cosh (\gamma b)+\gamma b \sinh (\gamma b)) L_{2}=0 \\
\gamma \sinh (\gamma b) L_{1}+(2 \nu \sinh (\gamma b)+\gamma b \cosh (\gamma b)) L_{2}=0 .
\end{array}\right.
$$

Its compatibility condition leads to the transcendental equation

$$
\sinh (2 \gamma b)+2 \gamma b=0
$$

which has only the trivial real root $\gamma=0$, therefore we will consider an infinite sequence of complexvalued roots $\gamma_{k}=\alpha_{k}+i \beta_{k}, k=1,2, \ldots$, where $\alpha_{k}$ and $\beta_{k}$ are real numbers, $i$ stands for imaginary unit.

Asymptotic formulas at $k \rightarrow \infty$ for real $\alpha_{k}$ and imaginary $\beta_{k}$ parts of the roots $\gamma_{k}$ can be easily obtained from the system

$$
\left\{\begin{array}{l}
\sinh \left(2 b \alpha_{k}\right) \cos \left(2 b \beta_{k}\right)+2 b \alpha_{k}=0 \\
\cosh \left(2 b \alpha_{k}\right) \sin \left(2 b \beta_{k}\right)+2 b \beta_{k}=0
\end{array}\right.
$$

following from (24). They are the next

$$
\alpha_{k}^{a}=\frac{1}{2} \ln (\pi+4 \pi k), \quad \beta_{k}^{a}=-\frac{\pi}{4}+\pi k .
$$

It is easy to see that, if $\gamma_{k}$ is a root of equation (24), then $-\gamma_{k}=-\alpha_{k}-i \beta_{k}, \bar{\gamma}_{k}=\alpha_{k}-i \beta_{k}$ and $-\bar{\gamma}_{k}=-\alpha_{k}+i \beta_{k}$ are also its roots. In accordance with this, we obtain four sequences of odd eigenfunctions of equation (19):

$$
\varphi_{k}(\zeta)=\kappa_{k} \sinh \left(\gamma_{k} \zeta\right)+\zeta \cosh \left(\gamma_{k} \zeta\right),
$$

where $\gamma_{k}$ and $\kappa_{k}$ are the roots of transcendental equation (24) and

$$
\kappa_{k}=(1-2 \nu) / \gamma_{k}-b \tanh \left(\gamma_{k} b\right) .
$$

Taking even axial function $\varphi(\zeta)$ in presentation (12), we come to other linear homogeneous system for the unknown coefficients in function (22):

$$
\left\{\begin{array}{l}
\gamma \sinh (\gamma b) L_{1}+((2 \nu-1) \sinh (\gamma b)+\gamma b \cosh (\gamma b)) L_{2}=0 \\
\gamma \cosh (\gamma b) L_{1}+(2 \nu \cosh (\gamma b)+\gamma b \sinh (\gamma b)) L_{2}=0
\end{array}\right.
$$

Compatibility condition of this system brings to the transcendental equation

$$
\sinh (2 \gamma b)-2 \gamma b=0
$$

This equation, as well as equation (24), has two pair of sequences of complex-conjugated roots $\gamma_{k}, \bar{\gamma}_{k}$ and $-\gamma_{k},-\bar{\gamma}_{k}, k=1,2, \ldots$ This enables to consider four sequences of even eigenfunctions of 
Table 1. Positive values of the real and imaginary parts of roots of equations (24) and (30).

\begin{tabular}{|c|c|c|c|c|}
\hline & \multicolumn{2}{|c|}{ Odd } & \multicolumn{2}{c|}{ Even } \\
\hline$k$ & $\alpha_{k}$ & $\beta_{k}$ & $\alpha_{k}$ & $\beta_{k}$ \\
\hline 1 & 1.02335 & 3.79089 & 0.74374 & 2.17518 \\
\hline 2 & 1.32419 & 6.97383 & 1.19683 & 5.38669 \\
\hline 3 & 1.50895 & 10.13593 & 1.42517 & 8.55631 \\
\hline 4 & 1.64319 & 13.29009 & 1.58059 & 11.71367 \\
\hline 5 & 1.74880 & 16.44027 & 1.69879 & 14.86554 \\
\hline 6 & 1.83590 & 19.58816 & 1.79425 & 18.01444 \\
\hline 7 & 1.91003 & 22.73459 & 1.87434 & 21.16153 \\
\hline 8 & 1.97457 & 25.88003 & 1.94335 & 24.30742 \\
\hline 9 & 2.03172 & 29.02476 & 2.00396 & 27.45247 \\
\hline 10 & 2.08300 & 32.16897 & 2.05802 & 30.59693 \\
\hline 11 & 2.12950 & 35.31278 & 2.10679 & 33.74092 \\
\hline 12 & 2.17204 & 38.45629 & 2.15122 & 36.88457 \\
\hline 13 & 2.21125 & 41.59954 & 2.19203 & 40.02794 \\
\hline 14 & 2.24760 & 44.74259 & 2.22975 & 43.17109 \\
\hline 15 & 2.28149 & 47.88547 & 2.26483 & 46.31405 \\
\hline 16 & 2.31322 & 51.02822 & 2.29761 & 49.45686 \\
\hline 17 & 2.34307 & 54.17085 & 2.32837 & 52.59955 \\
\hline 18 & 2.37123 & 57.31338 & 2.35734 & 55.74213 \\
\hline 19 & 2.39788 & 60.45583 & 2.38473 & 58.88462 \\
\hline 20 & 2.42319 & 63.59820 & 2.41070 & 62.02703 \\
\hline$\delta_{20}$ & 0.00007 & 0.0003 & 0.00051 & 0.0003 \\
\hline
\end{tabular}

equation (19):

$$
\varphi_{k}(\zeta)=\kappa_{k} \cosh \left(\gamma_{k} \zeta\right)+\zeta \sinh \left(\gamma_{k} \zeta\right)
$$

where $\gamma_{k}$ and $\kappa_{k}$ are the roots of transcendental equation (31) and

$$
\kappa_{k}=(1-2 \nu) / \gamma_{k}-b \operatorname{coth}\left(\gamma_{k} b\right) .
$$

Asymptotic solution of equation (31) at $k \rightarrow$ $\infty$ is

$$
\alpha_{k}^{a}=\frac{1}{2} \ln (\pi+4 \pi k), \quad \beta_{k}^{a}=\frac{\pi}{4}+\pi k .
$$

We solved transcendental equations (24), (30) numerically. The positive values of the real and imaginary parts of their first 20 roots are presented in Table 1. In the last row of the table the values of ratio error of the corresponding roots calculated with asymptotic formulas (26), (33) at $k=20$ are presented. The errors were calculated as

$$
\delta \alpha_{k}=\left|\tilde{\alpha}_{k}-\alpha_{k}\right| / \alpha_{k}, \quad \delta \beta_{k}=\left|\tilde{\beta}_{k}-\beta_{k}\right| / \beta_{k},
$$

where symbols with "tilde" $(\sim)$ mean the asymptotic values, whereas without it - the values determined by numerical solving of corresponding transcendental equation.

\subsection{The odd and even eigenfunctions}

Using function sequences (27) and (31), we can build the odd

$$
\chi_{k}^{(p)}(\xi, \zeta)=H_{0}^{(p)}\left(\gamma_{k} \xi\right)\left(\kappa_{k} \sinh \left(\gamma_{k} \zeta\right)+\zeta \cosh \left(\gamma_{k} \zeta\right)\right), \quad p=1,2
$$

and even

$$
\chi_{k}^{(p)}(\xi, \zeta)=H_{0}^{(p)}\left(\gamma_{k} \xi\right)\left(\kappa_{k} \cosh \left(\gamma_{k} \zeta\right)+\zeta \sinh \left(\gamma_{k} \zeta\right)\right), \quad p=1,2
$$

eigenfunctions of biharmonic problems for hollow cylinder with stress-free ends.

These functions identically satisfy equations (7) in the volume $\mathbb{V}$ and homogeneous boundary conditions (11) on the ends of the cylinder at any $k=1,2, \ldots$ and $p=1,2, \ldots$

\section{The systems of homogeneous solutions of axisymmetric elasticity problems for hollow cylinder}

Using obtained systems of eigenfunctions of biharmonic problems and formulas (13)-(18), one can obtain the systems of homogeneous solutions for corresponding elasticity problems.

In the case of symmetric problems they are

$$
\begin{aligned}
\sigma_{k}^{(p)}(\xi, \zeta)= & \frac{\gamma_{k}}{\xi}\left(\gamma_{k} \xi\left(2 \nu+1+\gamma_{k} \kappa_{k}\right) H_{0}^{(p)}\left(\gamma_{k} \xi\right)-\left(1+\gamma_{k} \kappa_{k}\right) H_{1}^{(p)}\left(\gamma_{k} \xi\right)\right) \sinh \left(\gamma_{k} \zeta\right) \\
& +\frac{\gamma_{k}^{2} \zeta}{\xi}\left(\gamma_{k} \xi H_{0}^{(p)}\left(\gamma_{k} \xi\right)-H_{1}^{(p)}\left(\gamma_{k} \xi\right)\right) \cosh \left(\gamma_{k} \zeta\right)
\end{aligned}
$$

Mathematical Modeling and Computing, Vol.7, No. 1, pp. 48-63 (2020) 


$$
\begin{aligned}
\tau_{k}^{(p)}(\xi, \zeta)= & \gamma_{k}^{2} H_{1}^{(p)}\left(\gamma_{k} \xi\right)\left(\left(2 \nu+\gamma_{k} \kappa_{k}\right) \sinh \left(\gamma_{k} \zeta\right)+\gamma_{k} \zeta \cosh \left(\gamma_{k} \zeta\right)\right) \\
\sigma_{z z k}^{(p)}(\xi, \zeta)= & -\left(\gamma_{k}\right)^{2} H_{0}^{(p)}\left(\gamma_{k} \xi\right)\left(\gamma_{k} \zeta \sinh \left(\gamma_{k} \zeta\right)+\left(2 \nu-1+\gamma_{k} \kappa_{k}\right) \cosh \left(\gamma_{k} \zeta\right)\right) \\
\sigma_{\theta \theta k}^{(p)}(\xi, \zeta)= & \frac{\gamma_{k}^{2}}{\xi} \zeta H_{1}^{(p)}\left(\gamma_{k} \xi\right) \sinh \left(\gamma_{k} \zeta\right) \\
& +\frac{\gamma_{k}}{\xi}\left(2 \nu \gamma_{k} \xi H_{0}^{(p)}\left(\gamma_{k} \xi\right)+\left(1+\gamma_{k} \kappa_{k}\right) H_{1}^{(p)}\left(\gamma_{k} \xi\right)\right) \cosh \left(\gamma_{k} \zeta\right), \\
u_{k}^{(p)}(\xi, \zeta)= & (1+\nu) \gamma_{k} H_{1}^{(p)}\left(\gamma_{k} \xi\right)\left(\gamma_{k} \zeta \sinh \left(\gamma_{k} \zeta\right)+\left(1+\gamma_{k} \kappa_{k}\right) \cosh \left(\gamma_{k} \zeta\right)\right) \\
v_{k}^{(p)}(\xi, \zeta)= & -(1+\nu) \gamma_{k} H_{0}^{(p)}\left(\gamma_{k} \xi\right)\left(\left(4 \nu-2+\gamma_{k} \kappa_{k}\right) \sinh \left(\gamma_{k} \zeta\right)+\gamma_{k} \zeta \cosh \left(\gamma_{k} \zeta\right)\right)
\end{aligned}
$$

Here $\xi \in\left[\xi_{\text {in }}, 1\right], \zeta \in[-b, b], p=1,2, \gamma_{k}, k=1,2, \ldots$ are the roots of transcendental equation (24), $\kappa_{k}$ are determined by formula (28).

For antisymmetric problems the homogeneous solutions have a look

$$
\begin{aligned}
\sigma_{k}^{(p)}(\xi, \zeta)= & \frac{\gamma_{k}}{\xi}\left(\gamma_{k} \xi\left(2 \nu+1+\gamma_{k} \kappa_{k}\right) H_{0}^{(p)}\left(\gamma_{k} \xi\right)-\left(1+\gamma_{k} \kappa_{k}\right) H_{1}^{(p)}\left(\gamma_{k} \xi\right)\right) \cosh \left(\gamma_{k} \zeta\right) \\
& +\frac{\gamma_{k}^{2} \zeta}{\xi}\left(\gamma_{k} \xi H_{0}^{(p)}\left(\gamma_{k} \xi\right)-H_{1}^{(p)}\left(\gamma_{k} \xi\right)\right) \sinh \left(\gamma_{k} \zeta\right) \\
\tau_{k}^{(p)}(\xi, \zeta)= & \gamma_{k}^{2} H_{1}^{(p)}\left(\gamma_{k} \xi\right)\left(\left(2 \nu+\gamma_{k} \kappa_{k}\right) \cosh \left(\gamma_{k} \zeta\right)+\gamma_{k} \zeta \sinh \left(\gamma_{k} \zeta\right)\right) \\
\sigma_{z z k}^{(p)}(\xi, \zeta)= & -\left(\gamma_{k}\right)^{2} H_{0}^{(p)}\left(\gamma_{k} \xi\right)\left(\gamma_{k} \zeta \cosh \left(\gamma_{k} \zeta\right)+\left(2 \nu-1+\gamma_{k} \kappa_{k}\right) \sinh \left(\gamma_{k} \zeta\right)\right) \\
\sigma_{\theta \theta k}^{(p)}(\xi, \zeta)= & \frac{\gamma_{k}^{2}}{\xi} \zeta H_{1}^{(p)}\left(\gamma_{k} \xi\right) \cosh \left(\gamma_{k} \zeta\right)+\frac{\gamma_{k}}{\xi}\left(2 \nu \gamma_{k} \xi H_{0}^{(p)}\left(\gamma_{k} \xi\right)+\left(1+\gamma_{k} \kappa_{k}\right) H_{1}^{(p)}\left(\gamma_{k} \xi\right)\right) \sinh \left(\gamma_{k} \zeta\right), \\
u_{k}^{(p)}(\xi, \zeta)= & (1+\nu) \gamma_{k} H_{1}^{(p)}\left(\gamma_{k} \xi\right)\left(\gamma_{k} \zeta \cosh \left(\gamma_{k} \zeta\right)+\left(1+\gamma_{k} \kappa_{k}\right) \sinh \left(\gamma_{k} \zeta\right)\right) \\
v_{k}^{(p)}(\xi, \zeta)= & -(1+\nu) \gamma_{k} H_{0}^{(p)}\left(\gamma_{k} \xi\right)\left(\left(4 \nu-2+\gamma_{k} \kappa_{k}\right) \cosh \left(\gamma_{k} \zeta\right)+\gamma_{k} \zeta \sinh \left(\gamma_{k} \zeta\right)\right)
\end{aligned}
$$

Here $\xi \in\left[\xi_{\text {in }}, 1\right], \zeta \in[-b, b], p=1,2, \gamma_{k}, k=1,2, \ldots$ are the roots of transcendental equation $(31), \kappa_{k}$ are determined by formula (33).

Each homogeneous solution $\left\{\sigma_{k}^{(p)}(\xi, \zeta), \tau_{k}^{(p)}(\xi, \zeta), \sigma_{z z k}^{(p)}(\xi, \zeta), \sigma_{\theta \theta k}^{(p)}(\xi, \zeta), u_{k}^{(p)}(\xi, \zeta), v_{k}^{(p)}(\xi, \zeta)\right\}$ identically satisfy equations (3)-(6) in volume $\mathbb{V}$ and homogeneous boundary conditions (2) on the ends of cylinder.

\section{Solution representation}

We represent the Love function as a series expansion in terms of eigenfunctions (35) and (36). As the eigenfunctions are complex-valued, to obtain the real Love function $\chi(\xi, \zeta)$, we take it in the form

$$
\chi(\xi, \zeta)=\frac{1}{2} \sum_{k=1}^{\infty} \sum_{\lambda=1}^{4} C_{k}^{\mu} \chi_{k}^{\mu}(\xi, \zeta) .
$$

Here we use the denotations: $C_{1}^{1}$ and $C_{k}^{2}$ are undefined complex constants, $\chi_{k}^{1}(\xi, \zeta)=\chi_{k}^{(1)}(\xi, \zeta)$, $\chi_{k}^{2}(\xi, \zeta)=\chi_{k}^{(2)}(\xi, \zeta), \chi_{k}^{3}(\xi, \zeta)=\bar{\chi}_{k}^{(1)}(\xi, \zeta), \chi_{k}^{4}(\xi, \zeta)=\bar{\chi}_{k}^{(2)}(\xi, \zeta), C_{k}^{3}=\bar{C}_{k}^{1}, C_{k}^{4}=\bar{C}_{k}^{2}$, the symbols with overline denote corresponding complex conjugate values.

In the case of an even problem we take in the right hand part of equation (49) the odd eigenfunctions (35), in the other case - the even eigenfunctions (36).

We represent the solution of problem (7), (10), (11) for both symmetric and antisymmetric cases in the form 


$$
\begin{aligned}
\sigma & =\frac{\partial}{\partial \zeta}\left(\nu \nabla^{2} \chi-\frac{\partial^{2} \chi}{\partial \xi^{2}}\right)+\sigma^{0}, & \tau & =\frac{\partial}{\partial \xi}\left((1-\nu) \nabla^{2} \chi-\frac{\partial^{2} \chi}{\partial \zeta^{2}}\right)+\tau^{0}, \\
\sigma_{z z} & =\frac{\partial}{\partial \zeta}\left((2-\nu) \nabla^{2} \chi-\frac{\partial^{2} \chi}{\partial \zeta^{2}}\right), & \sigma_{\theta \theta} & =\frac{\partial}{\partial \zeta}\left(\nu \nabla^{2} \chi-\frac{1}{\xi} \frac{\partial \chi}{\partial \xi}\right)+\sigma_{\theta \theta}^{0} . \\
u & =-(1+\nu) \frac{\partial^{2} \chi}{\partial \xi \partial \zeta}+u^{0}, & v & =2\left(1-\nu^{2}\right) \nabla^{2} \chi-(1+\nu) \frac{\partial^{2} \chi}{\partial \zeta^{2}}+v^{0} .
\end{aligned}
$$

Here $\chi$ is the Love function (49) and the functions $\sigma^{0}, \tau^{0}, \sigma_{\theta \theta}^{0}$ have the form

$$
\sigma^{0}=\frac{1}{\xi^{2}} c_{1}+c_{2}, \quad \tau^{0}=\frac{c_{3}}{\xi}, \quad \sigma_{\theta \theta}^{0}=-c_{1} \frac{1}{\xi^{2}}+c_{2} .
$$

The function $u^{0}$ and $v^{0}$ are determined follow on the base of functions from (52) with the use of relations (4) and (5):

$$
u^{0}=-\frac{c_{1}}{2 G \xi}+\frac{c_{2} \xi}{2 G(1+\nu)}, \quad v^{0}=-c_{2} \frac{\nu \zeta}{2 G(1+\nu)}+2 c_{3}(1+\nu) \ln \xi .
$$

In formulas (52), (53) $c_{1}, c_{2}, c_{3}$ are undefined real constants: for the case of symmetry $c_{3} \equiv 0$, whereas $c_{1}=c_{2} \equiv 0$ for antisymmetry.

It is easy to verify that the strain-stressed state determined by functions $\sigma^{0}, \tau^{0}, \sigma_{\theta \theta}^{0}, \sigma_{z z}^{0} \equiv 0$ satisfy the equation of equilibrium (3) and the compatibility equations in stresses (Beltrami-Mitchell equations [26]).

Solution Presentation (50), (51) identically satisfies the equations (3)-(6) in the body volume $\mathbb{V}$ and homogeneous boundary conditions (2) on its the cylinder bases for arbitrary values of the coefficients in Love function series expansion (49).

\section{The variational method of homogeneous solutions}

We subordinate the solutions (49) to the boundary conditions (10) in the quadratic norm $L_{2}$, introducing functional

$$
\begin{aligned}
F= & \int_{0}^{b}\left[\left(\left.\sigma\right|_{\xi=\xi_{\text {in }}}-\sigma_{\text {in }}(\zeta)\right)^{2}+\left(\left.\tau\right|_{\xi=\xi_{\text {in }}}-\tau_{\text {in }}(\zeta)\right)^{2}\right] d \zeta \\
& +\int_{0}^{b}\left[\left(\left.\sigma\right|_{\xi=1}-\sigma_{\text {out }}(\zeta)\right)^{2}+\left(\left.\tau\right|_{\xi=1}-\tau_{\text {out }}(\zeta)\right)^{2}\right] d \zeta .
\end{aligned}
$$

Substituting into the functional presentation (50) and applying to it the necessary conditions of the minimum

$$
\frac{\partial F}{\partial C_{m}^{\lambda}}=0, \quad \frac{\partial F}{\partial c_{j}}=0, \quad m=1,2, \ldots, \quad \lambda=1, \ldots, 4, \quad j=1, \ldots, 4
$$

we come to the linear algebraic system

$$
\sum_{k=1}^{\infty} \sum_{\mu=1}^{4} M_{m k}^{\lambda \mu} C_{k}^{\mu}=K_{m}^{\lambda}, \quad m=1,2, \ldots, \quad \lambda=1, \ldots, 4 .
$$

Its coefficients for symmetric problems are determined as

$$
\begin{aligned}
M_{m k}^{\lambda \mu}= & \frac{1}{2} \int_{0}^{b}\left(\sigma_{m}^{\lambda}\left(\xi_{\text {in }}, \zeta\right) \sigma_{k}^{\mu}\left(\xi_{\text {in }}, \zeta\right)+\tau_{m}^{\lambda}\left(\xi_{\text {in }}, \zeta\right) \tau_{k}^{\mu}\left(\xi_{\text {in }}, \zeta\right)\right) d \zeta \\
& +\frac{1}{2} \int_{0}^{b}\left(\sigma_{m}^{\lambda}(1, \zeta) \sigma_{k}^{\mu}(1, \zeta)+\tau_{m}^{\lambda}(1, \zeta) \tau_{k}^{\mu}(1, \zeta)\right) d \zeta
\end{aligned}
$$

Mathematical Modeling and Computing, Vol.7, No. 1, pp. 48-63 (2020) 


$$
\begin{aligned}
& -\frac{1}{2 b}\left(\int_{0}^{b} \sigma_{m}^{\lambda}\left(\xi_{\text {in }}, \zeta\right) d \zeta \int_{0}^{b} \sigma_{k}^{\mu}\left(\xi_{\text {in }}, \zeta\right) d \zeta+\int_{0}^{b} \sigma_{m}^{\lambda}(1, \zeta) d \zeta \int_{0}^{b} \sigma_{k}^{\mu}(1, \zeta) d \zeta\right), \\
K_{m}^{\lambda}= & \int_{0}^{b}\left(\sigma_{\text {in }}(\zeta) \sigma_{m}^{\lambda}\left(\xi_{\text {in }}, \zeta\right)+\tau_{\text {in }}(\zeta) \tau_{m}^{\lambda}\left(\xi_{\text {in }}, \zeta\right)\right) d \zeta \\
& +\int_{0}^{b}\left(\sigma_{\text {out }}(\zeta) \sigma_{m}^{\lambda}(1, \zeta)+\tau_{\text {out }}(\zeta) \tau_{m}^{\lambda}(1, \zeta)\right) d \zeta \\
& -\frac{1}{b}\left(\int_{0}^{b} \sigma_{\text {in }}(\zeta) d \zeta \int_{0}^{b} \sigma_{m}^{\lambda}\left(\xi_{\text {in }}, \zeta\right) d \zeta+\int_{0}^{b} \sigma_{\text {out }}(\zeta) d \zeta \int_{0}^{b} \sigma_{m}^{\lambda}(1, \zeta) d \zeta\right) .
\end{aligned}
$$

Functions $\sigma_{k}^{\mu}(\xi, \zeta), \tau_{k}^{\mu}(\xi, \zeta)$ in these formulas are calculated by formulas (37), (38), in which $\gamma_{k}$ are roots of equation (24) and constants $\kappa_{k}$ are defined by formula (28).

In the case of antisymmetry, the coefficients of system (55) have the look

$$
\begin{aligned}
M_{m k}^{\lambda \mu}= & \frac{1}{2} \int_{0}^{b}\left(\sigma_{m}^{\lambda}\left(\xi_{\text {in }}, \zeta\right) \sigma_{k}^{\mu}\left(\xi_{\text {in }}, \zeta\right)+\tau_{m}^{\lambda}\left(\xi_{\text {in }}, \zeta\right) \tau_{k}^{\mu}\left(\xi_{\text {in }}, \zeta\right)\right) d \zeta \\
& +\frac{1}{2} \int_{0}^{b}\left(\sigma_{m}^{\lambda}(1, \zeta) \sigma_{k}^{\mu}(1, \zeta)+\tau_{m}^{\lambda}(1, \zeta) \tau_{k}^{\mu}(1, \zeta)\right) d \zeta \\
& -\frac{3}{2 b^{3}}\left(\int_{0}^{b} \sigma_{m}^{\lambda}\left(\xi_{\text {in }}, \zeta\right) d \zeta \int_{0}^{b} \sigma_{k}^{\mu}\left(\xi_{\text {in }}, \zeta\right) d \zeta+\int_{0}^{b} \sigma_{m}^{\lambda}(1, \zeta) d \zeta \int_{0}^{b} \sigma_{k}^{\mu}(1, \zeta) d \zeta\right), \\
K_{m}^{\lambda}= & \int_{0}^{b}\left(\sigma_{\text {in }}(\zeta) \sigma_{m}^{\lambda}\left(\xi_{\text {in }}, \zeta\right)+\tau_{\text {in }}(\zeta) \tau_{m}^{\lambda}\left(\xi_{\text {in }}, \zeta\right)\right) d \zeta \\
& +\int_{0}^{b}\left(\sigma_{\text {out }}(\zeta) \sigma_{m}^{\lambda}(1, \zeta)+\tau_{\text {out }}(\zeta) \tau_{m}^{\lambda}(1, \zeta)\right) d \zeta \\
& -\frac{3}{b^{3}}\left(\int_{0}^{b} \sigma_{\text {in }}(\zeta) d \zeta \int_{0}^{b} \sigma_{m}^{\lambda}\left(\xi_{\text {in }}, \zeta\right) d \zeta+\int_{0}^{b} \sigma_{\text {out }}(\zeta) d \zeta \int_{0}^{b} \sigma_{m}^{\lambda}(1, \zeta) d \zeta\right) .
\end{aligned}
$$

Functions $\sigma_{k}^{\mu}(\xi, \zeta), \tau_{k}^{\mu}(\xi, \zeta)$ in these formulas are calculated by formulas (43), (44), in which $\gamma_{k}$ are roots of equation (30) and constants $\kappa_{k}$ are defined by formula (32).

We used the following notations in the formulas (56)-(59):

$$
\begin{aligned}
& \sigma_{k}^{1}(\xi, \zeta)=\sigma_{k}^{(1)}(\xi, \zeta), \quad \sigma_{k}^{2}(\xi, \zeta)=\sigma_{k}^{(2)}(\xi, \zeta), \quad \sigma_{k}^{3}(\xi, \zeta)=\bar{\sigma}_{k}^{(1)}(\xi, \zeta), \quad \sigma_{k}^{4}(\xi, \zeta)=\bar{\sigma}_{k}^{(2)}(\xi, \zeta), \\
& \tau_{k}^{1}(\xi, \zeta)=\tau_{k}^{(1)}(\xi, \zeta), \quad \tau_{k}^{2}(\xi, \zeta)=\tau_{k}^{(2)}(\xi, \zeta), \quad \tau_{k}^{3}(\xi, \zeta)=\bar{\tau}_{k}^{(1)}(\xi, \zeta), \quad \tau_{k}^{4}(\xi, \zeta)=\bar{\tau}_{k}^{(2)}(\xi, \zeta) .
\end{aligned}
$$

The constants $c_{j}$ are determined as

$$
\begin{aligned}
& c_{1}=-\frac{\xi_{\text {in }}^{2}}{b\left(1-\xi_{\text {in }}^{2}\right)} \int_{0}^{b}\left(\frac{1}{2} \sum_{k=1}^{\infty} \sum_{\mu=1}^{4} C_{k}^{\mu}\left(\sigma_{k}^{\mu}\left(\xi_{\text {in }}, \zeta\right)-\sigma_{k}^{\mu}(1, \zeta)\right)-\sigma_{\text {in }}(\zeta)+\sigma_{\text {out }}(\zeta)\right) d \zeta \\
& c_{2}=\frac{1}{b\left(1-\xi_{\text {in }}^{2}\right)} \int_{0}^{b}\left(\frac{1}{2} \sum_{k=1}^{\infty} \sum_{\mu=1}^{4} C_{k}^{\mu}\left(\xi_{\text {in }}^{2} \sigma_{k}^{\mu}\left(\xi_{\text {in }}, \zeta\right)-\sigma_{k}^{\mu}(1, \zeta)\right)-\xi_{\text {in }}^{2} \sigma_{\text {in }}(\zeta)+\sigma_{\text {out }}(\zeta)\right) d \zeta \\
& c_{3}=-\frac{3 \xi_{\text {in }}^{2}}{b^{3}\left(1-\xi_{\text {in }}^{2}\right)} \int_{0}^{b}\left(\frac{1}{2} \sum_{k=1}^{\infty} \sum_{\mu=1}^{4} C_{k}^{\mu}\left(\sigma_{k}^{\mu}\left(\xi_{\text {in }}, \zeta\right)-\sigma_{k}^{\mu}(1, \zeta)\right)-\sigma_{\text {in }}(\zeta)+\sigma_{\text {out }}(\zeta)\right) d \zeta \\
& c_{4}=\frac{3}{b^{3}\left(1-\xi_{\text {in }}^{2}\right)} \int_{0}^{b}\left(\frac{1}{2} \sum_{k=1}^{\infty} \sum_{\mu=1}^{4} C_{k}^{\mu}\left(\xi_{\text {in }}^{2} \sigma_{k}^{\mu}\left(\xi_{\text {in }}, \zeta\right)-\sigma_{k}^{\mu}(1, \zeta)\right)-\xi_{\text {in }}^{2} \sigma_{\text {in }}(\zeta)+\sigma_{\text {out }}(\zeta)\right) d \zeta
\end{aligned}
$$




\section{Numerical study of the problem solutions}

We solved the system (55) with the use of reduction method, keeping in the expansion (49) a finite number $N$ of terms. This leads to the linear $4 N \times 4 N$ system of algebraic equations in the form:

$$
\sum_{k=1}^{N} \sum_{\mu=1}^{4} M_{m k}^{\lambda \mu} C_{k}^{\mu}=K_{m}^{\lambda}, \quad m=1,2, \ldots, N, \quad \lambda=1, \ldots, 4 .
$$

To evaluate numerically the convergence of reduction method, we took the functions of right-hand sides in the boundary conditions (10) for symmetric and antisymmetric cases in forms (61) and (62) respectively:

and

$$
\begin{aligned}
\sigma_{\text {in }}(\zeta) & =-0.7 \sigma_{0} \exp \left(-\frac{\zeta^{2}}{d_{1}^{2}}\right) \\
\tau_{\text {in }}(\zeta) & =0.7 \tau_{0} \exp \left(\frac{-\left(\zeta-0.8 \zeta_{0}\right)^{2}}{d_{1}^{2}}\right)-0.7 \tau_{0} \exp \left(\frac{-\left(\zeta+0.8 \zeta_{0}\right)^{2}}{d_{1}^{2}}\right) \\
\sigma_{\text {out }}(\zeta) & =\sigma_{0} \exp \left(\frac{-\left(\zeta-\zeta_{0}\right)^{2}}{d_{2}^{2}}\right)+\sigma_{0} \exp \left(\frac{-\left(\zeta+\zeta_{0}\right)^{2}}{d_{2}^{2}}\right) \\
\tau_{\text {out }}(\zeta) & =-\tau_{0} \exp \left(\frac{-\left(\zeta-\zeta_{0}\right)^{2}}{d_{2}^{2}}\right)+\tau_{0} \exp \left(\frac{-\left(\zeta+\zeta_{0}\right)^{2}}{d_{2}^{2}}\right)
\end{aligned}
$$

$$
\begin{aligned}
\sigma_{\text {in }}(\zeta) & =0.7 \tau_{0} \exp \left(\frac{-\left(\zeta-0.8 \zeta_{0}\right)^{2}}{d_{1}^{2}}\right)-0.7 \tau_{0} \exp \left(\frac{-\left(\zeta+0.8 \zeta_{0}\right)^{2}}{d_{1}^{2}}\right), \\
\tau_{\text {in }}(\zeta) & =-0.7 \sigma_{0} \exp \left(-\frac{\zeta^{2}}{d_{1}^{2}}\right) \\
\sigma_{\text {out }}(\zeta) & =-\sigma_{0} \exp \left(\frac{-\left(\zeta-\zeta_{0}\right)^{2}}{d_{2}^{2}}\right)+\sigma_{0} \exp \left(\frac{-\left(\zeta+\zeta_{0}\right)^{2}}{d_{2}^{2}}\right) \\
\tau_{\text {out }}(\zeta) & =\tau_{0} \exp \left(\frac{-\left(\zeta-\zeta_{0}\right)^{2}}{d_{2}^{2}}\right)+\tau_{0} \exp \left(\frac{-\left(\zeta+\zeta_{0}\right)^{2}}{d_{2}^{2}}\right)
\end{aligned}
$$

Errors for the obtained solutions were evaluated by the values of corresponding functionals as:

$$
\delta_{L_{2}}=\left(F^{N}\right)^{1 / 2} / 2
$$

The errors of solutions, obtained at $\sigma_{0}=0.05, \tau_{0}=0.05, \nu=0.25, \xi_{\text {in }}=0.4, \zeta_{0}=0.5 b, d_{1}=0.15 b$, $d_{2}=0.1 b, b=2$ for different number $N$ in the cases of problem symmetry and antisymmetry are given in Table 2 .

Table 2. Reduction errors.

\begin{tabular}{|c|c|c|c|c|c|c|c|}
\hline$N$ & 3 & 5 & 7 & 9 & 11 & 13 & 15 \\
\hline$\delta_{L_{2}}$, symmetric & 0.15504 & 0.08583 & 0.04294 & 0.01762 & 0.00665 & 0.00201 & 0.00067 \\
\hline$\delta_{L_{2}}$, antisymmetric & 0.15365 & 0.07521 & 0.03776 & 0.01754 & 0.00647 & 0.00203 & 0.00071 \\
\hline
\end{tabular}

The plots of dimensionless stress components $\sigma / \sigma_{0}, \tau / \tau_{0}, \sigma_{\theta \theta} / \sigma_{0}$ and $\sigma_{z z} / \sigma_{0}$ as functions of axial coordinate, calculated at various values of the radial coordinate $\xi \in\{0.4,0.55,0.75,1\}$ (curves 1 to 4 correspondingly) for the symmetric problem are shown in Figs. 1-4.

Plots of the same dimensionless stress components as functions of axial coordinate, calculated at $\xi \in\{0.4,0.55,0.75,1\}$ (curves 1 to 4 correspondingly) for the antisymmetric problem are shown in Figs. $5-8$. 


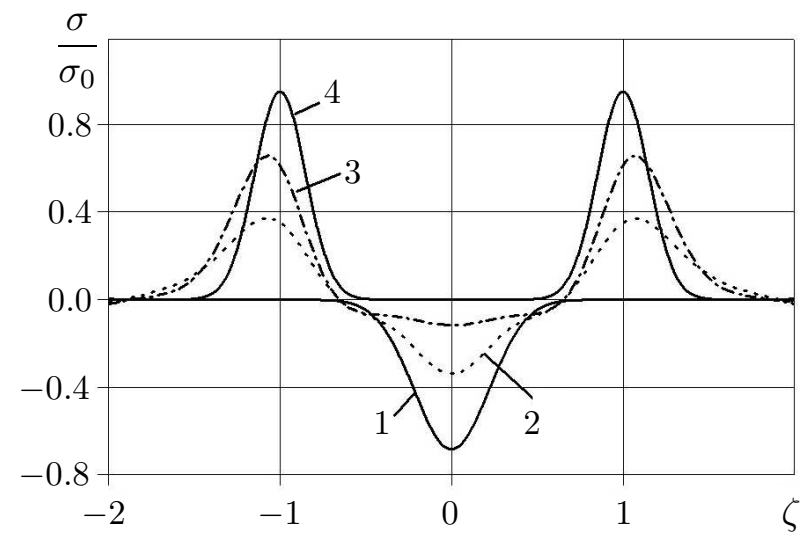

Fig. 1. Axial distributions of the normal stress component at various values of radial coordinate.

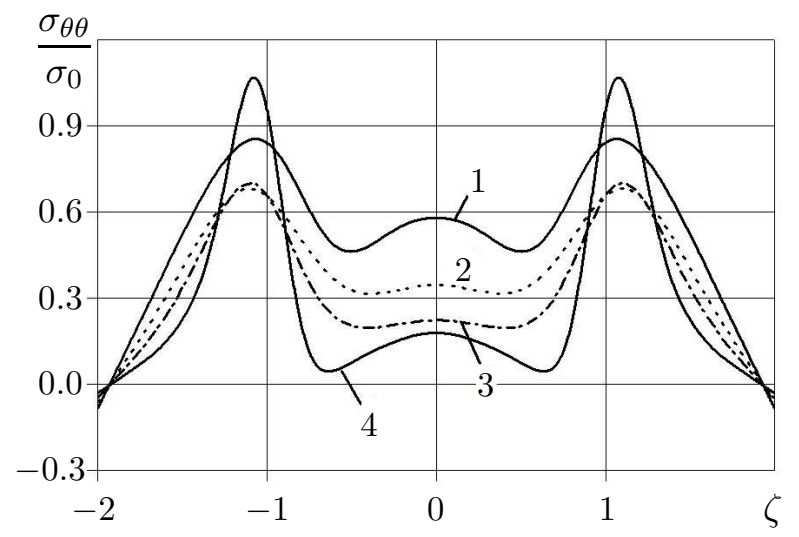

Fig. 3. Axial distributions of the hoop stress component at various values of radial coordinate.

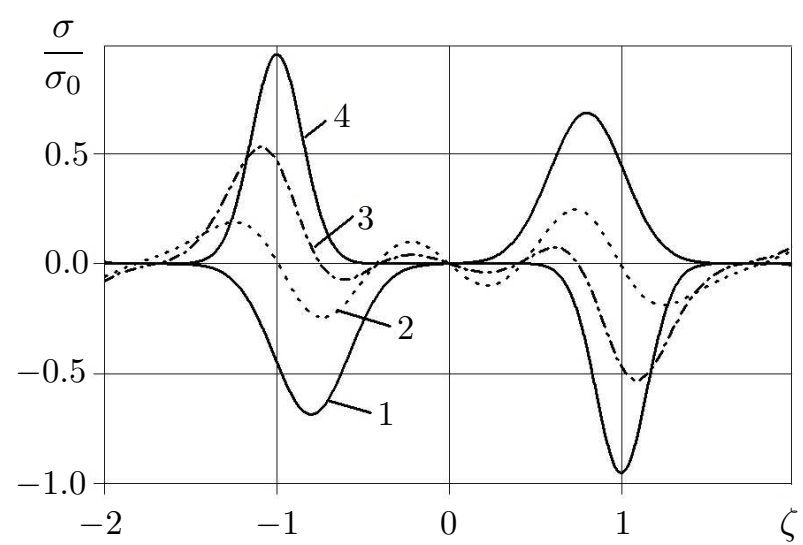

Fig. 5. Axial distributions of the normal stress component at various values of radial coordinate.

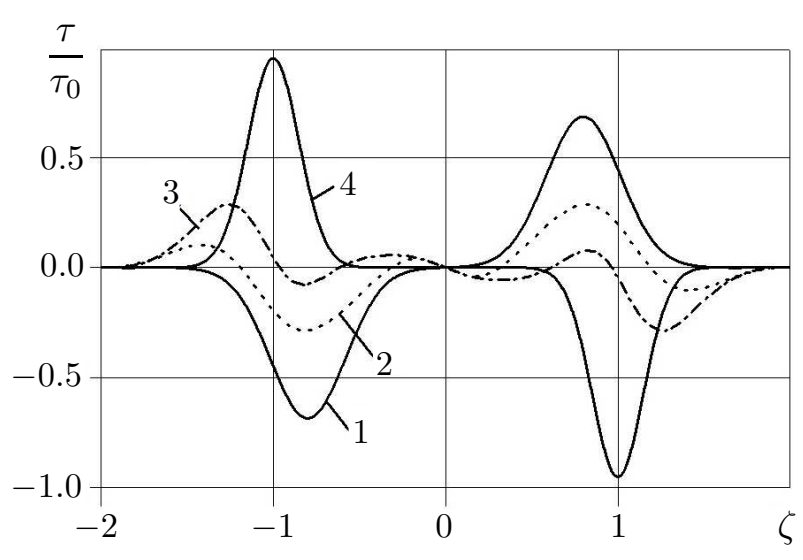

Fig. 2. Axial distributions of the tangential stress component at various values of radial coordinate.

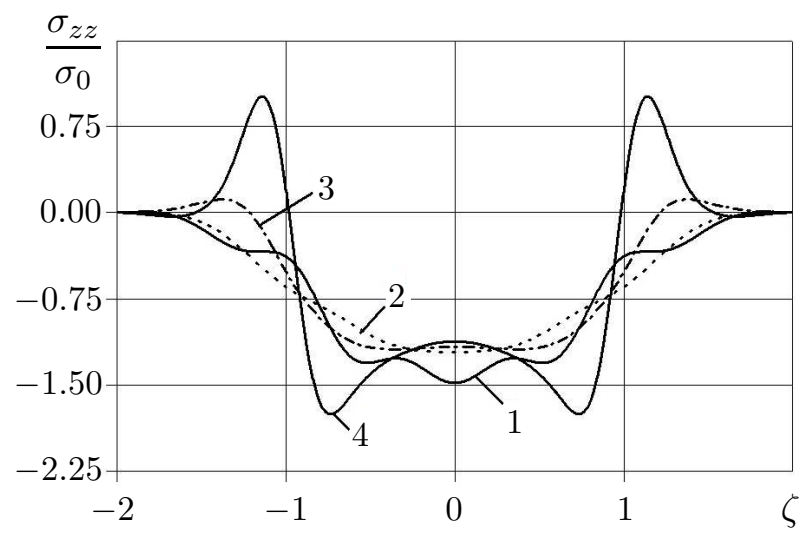

Fig. 4. Axial distributions of the axial stress component at various values of radial coordinate.

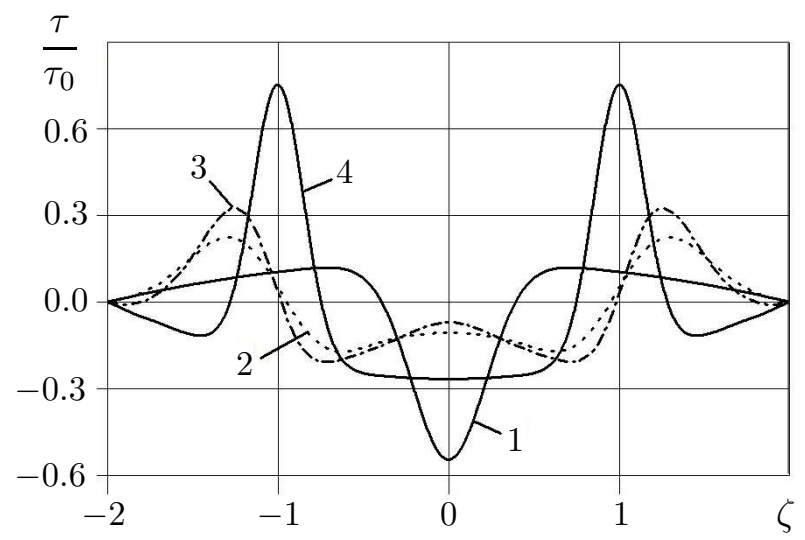

Fig. 6. Axial distributions of the tangential stress component at various values of radial coordinate.

All the curves presented in Figs. 1-8 were calculated at $N=15$. As one can see, if the boundary data are sufficiently smooth functions, the graphs of stress components $\sigma_{r r}$ and $\sigma_{r z}$ on the inner and outer surfaces (curves 1 and 4 in Figs. 1, 2, and 5,6) coincide with the corresponding graphs of the prescribed on these surfaces normal $\sigma$ and tangential $\tau$ traction.

To study how the method behaves when the boundary data in conditions (10) are high-gradient functions, we solved two problems for the case of symmetry problem, using as boundary data functions (64) and (65), and another two problems for the case of antisymmetry with boundary functions 
in forms (66) and (67):

$$
\begin{aligned}
& \sigma_{\text {in }}(\zeta)=0, \quad \tau_{\text {in }}(\zeta)=0, \quad \sigma_{\text {out }}(\zeta)=\sigma_{0}\left\{\begin{array}{cc}
0.5, & |\zeta|<1, \\
-1, & 1 \leqslant|\zeta| \leqslant 1.5, \\
0, & |\zeta|>1.5,
\end{array} \quad \tau_{\text {out }}(\zeta)=0\right. \\
& \sigma_{\text {in }}(\zeta)=0, \quad \tau_{\text {in }}(\zeta)=0, \quad \sigma_{\text {out }}(\zeta)=0, \quad \tau_{\text {out }}(\zeta)=\tau_{0}\left\{\begin{array}{cc}
0, & |\zeta|<1, \\
1, & 1 \leqslant|\zeta| \leqslant 1.5, \\
-1, & -1.5 \leqslant|\zeta| \leqslant-1, \\
0, & |\zeta|>1.5
\end{array}\right. \\
& \sigma_{\text {in }}(\zeta)=0, \quad \tau_{\text {in }}(\zeta)=0, \quad \sigma_{\text {out }}(\zeta)=0, \quad \tau_{\text {out }}(\zeta)=\tau_{0}\left\{\begin{array}{cc}
0.5, & |\zeta|<1 \\
-1, & 1 \leqslant|\zeta| \leqslant 1.5 \\
0, & |\zeta|>1.5
\end{array}\right. \\
& \sigma_{\text {in }}(\zeta)=0, \quad \tau_{\text {in }}(\zeta)=0, \quad \sigma_{\text {out }}(\zeta)=\sigma_{0}\left\{\begin{array}{cc}
0.5, & \zeta<0, \\
-0.5, & \zeta \geqslant 0,
\end{array} \tau_{\text {out }}(\zeta)=0 .\right.
\end{aligned}
$$

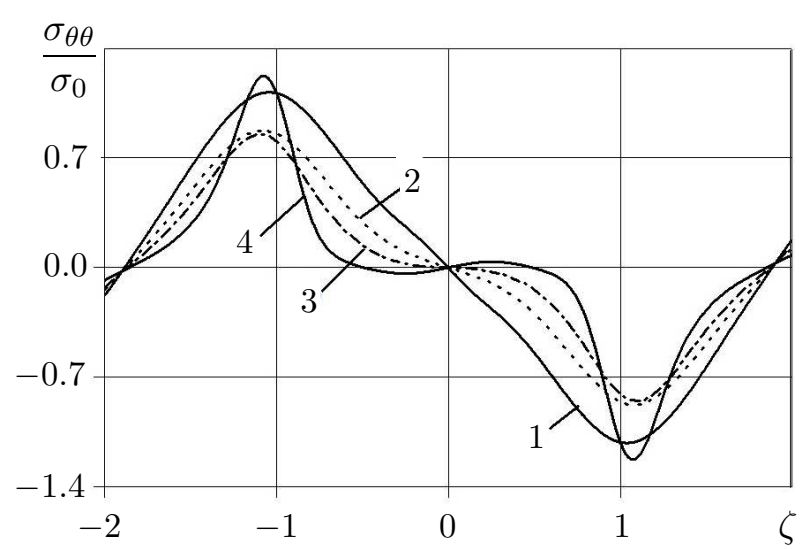

Fig. 7. Axial distributions of the hoop stress component at various values of radial coordinate.

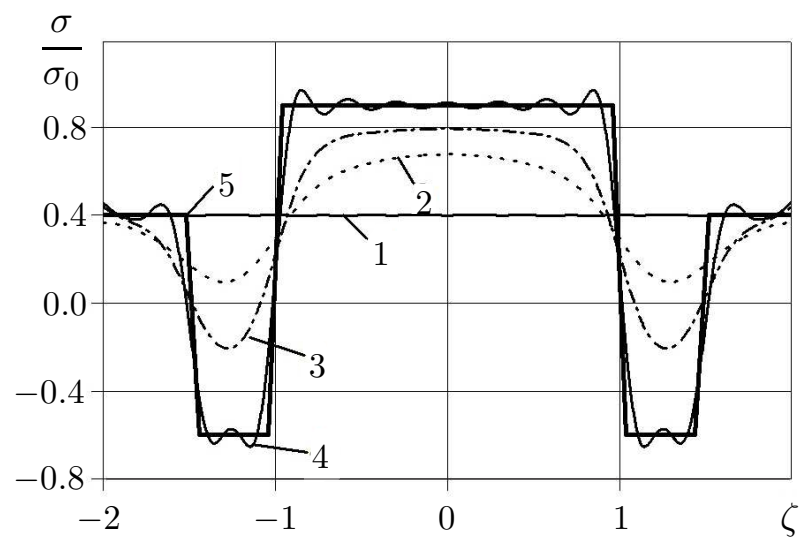

Fig. 9. Axial distributions of the normal stress component for solution, obtained at boundary functions (64).

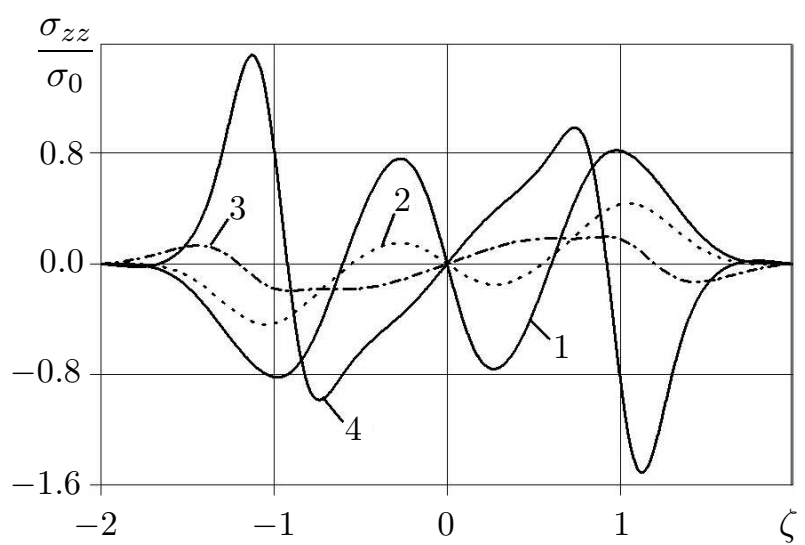

Fig. 8. Axial distributions of the axial stress component at various values of radial coordinate.

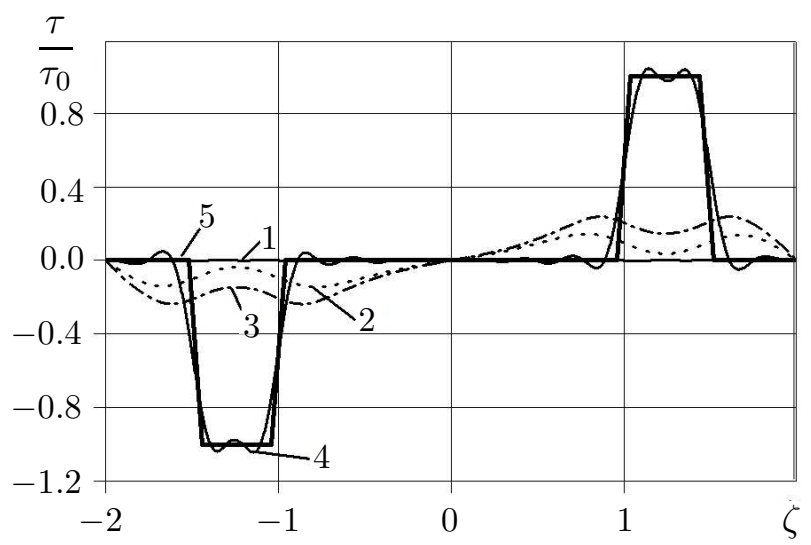

Fig. 10. Axial distributions of the tangential stress component for solution, obtained at boundary functions (65).

In Figs. 9 and 10 axial dependencies of the non-dimensional normal $\sigma / \sigma_{0}$ and tangential $\tau / \tau_{0}$ stress components calculated for $\xi \in\{0.4,0.55,0.75,1\}$ (curves 1-4) with the use of solutions, obtained for boundary functions in forms (64) and (65) correspondingly are shown. Curves 5 correspond the functions prescribed on the boundary. The solutions were obtained for $N=15$.

Similar plots for antisymmetric problems with boundary functions (66), (67) are presented in Figs. 11, 12. 


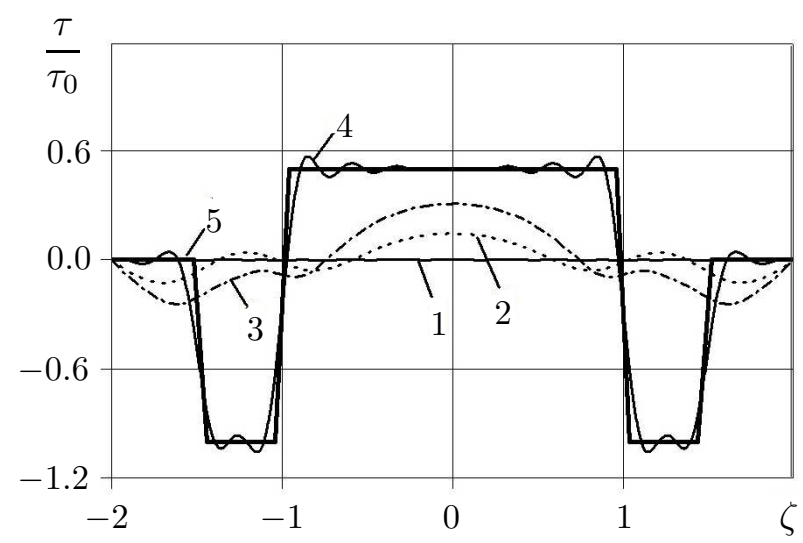

Fig. 11. Axial distributions of the normal stress component for solution, obtained at boundary functions (66).

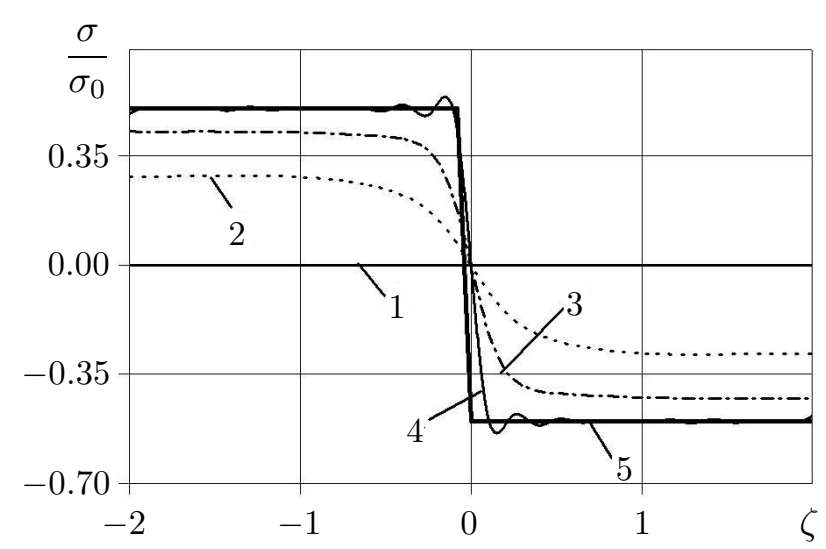

Fig. 12. Axial distributions of the tangential stress component for solution, obtained at boundary functions (67).

As we can see, even for discontinuous boundary functions obtained numerical solutions are rather precise - the reductions errors for both symmetric and antistmmertic problems do not exceed $6 \cdot 10^{-3} \%$.

\section{Conclusions}

The hollow cylinder is a three-dimensional body, bounded by two systems of surfaces: the lateral surface boundary is formed by two coaxial cylindrical surfaces, and the bases are two plane areas. Using the principle of superposition, the problems of the theory of elasticity for such bodies can be reduced to a sequence of two problems: in the first case, the homogeneous boundary conditions are given on a system of the lateral surfaces boundary, and in the second case - they are given on another one of the cylinder's bases. If the lateral surface is formed by coaxial circular cylinders, and the bases are orthogonal to them, then, under the appropriate boundary conditions, one can consider the axially symmetric statement for both of these cases. Using the Love function, axisymmetric problems of the theory of elasticity for a straight circular cylinder can be reduced to a biharmonic equation in cylindrical coordinates with the corresponding boundary conditions.

We have considered the elasticity problem for the straight hollow cylinder, on the basis of which homogeneous boundary conditions in stresses are given. Applying to this problem the method of variables separation, according to which the biharmonic problem solution is presented as the product of axial and radial functions, we came to a homogeneous boundary value problem for the ordinary differential equation regarding the axial function. Using the eigenfunctions of this problem, we constructed a system of homogeneous solutions of an axially symmetric elasticity problem for the hollow cylinder with unloaded bases. In fact, the solution of the biharmonic problem was represented by its development in terms of the obtained systems of homogeneous solutions.

This representation depends on the four infinite sequences of real constants, which can be used to subordinate the solution to four boundary conditions, prescribed on the lateral boundary. We have applied for that the variational method, which was previously developed for plane elasticity problems in a rectangular domain, as well as for a solid cylinder. Under this method subordination the solution to the boundary condition is carried out not pointwise, but "in average", i.e. in the norm $L_{2}$. For this purpose, a functional, which determines the mean square deviation of the problem solution from all boundary functions, prescribed on the lateral surface, was introduced. Applying the necessary minimum conditions to this functional, we obtain the infinite system of algebraic equations with respect to series expansion coefficients $C_{k}^{\mu}$.

The system was solved applying the reduction method. By this method the representation of biharmonic equation solution is restricted by first $N$ number of terms in its series development, what leads to a finite system of algebraic equations of size $N \times N$. 
The carried out numerical studies confirmed the good convergence of the solution with growth of $N$ value. The reduction error, calculated from the value of target functional at $N=15$ for continuous boundary functions, does not exceed $7 \cdot 10^{-5} \%$. The study of the solution convergence for problems with boundary functions containing jump discontinuity (piecewise homogeneous) also gave a good result the reduction error, calculated by the value of the target functional, is of order $6 \cdot 10^{-3} \%$.

[1] Collin F., Caillerie D., Chambon R. Analytical solutions for the thick-walled cylinder problem modeledwith an isotropic elastic second gradient constitutive equation. International Journal of Solids and Structures. 46 (22-23), 3927-3937 (2009).

[2] Zang W. The Elastic Solution of a Radial Heterogeneous Cylinder Subjected to Non-Uniform Distributed Normal and Tangential Loads. Mathematical Problems in Engineering. 2018, Article ID 9230609, 8 p. (2018).

[3] Chatzigeorgiou G., Charalambakis N., Murat F. Homogenization problems of a hollow cylinder made of elastic materials with discontinuous properties. International Journal of Solids and Structures. 45 (1819), 5165-5180 (2008).

[4] Theotokoglou E. E., Stampouloglou I. H. The radially nonhomogeneous elastic axisymmentric problem. International Journal of Solids and Structures. 45 (25-26), 6535-6552 (2008).

[5] Avci A., Bulu A., Yapici A. Axisymmetric smooth contact for an elastic isotropic infinite hollow cylinder compressed by an outer rigid ring with circular profile. Acta Mechanica Sinica. 22 (1), 46-53 (2006).

[6] Tokovyy Yu. V., Ma Chien-Ching. Analysis of residual stresses in a long hollow cylinder. International Journal of Pressure Vessels and Piping. 88 (5-7), 248-255 (2011).

[7] Ramesh M., Kailas S. V., Simha K. R. Y. Axisymmetric fretting analysis in coated cylinder. Sadhana. 33, 299-327 (2008).

[8] Grinchenko V. T. The axisymmetric problem of the theory of elasticity for a thick-walled cylinder of finite length. Soviet Applied Mechanics. 3 (8), 65-70 (1967).

[9] Bazarenko N. A. The contact problem for hollow and solid cylinders with stress-free faces. Journal of Applied Mathematics and Mechanics. 72, 214-225 (2008).

[10] Lurie S. A., Vasiliev V. V. The biharmonic problem in the theory of elasticity. Gordon and Breach, Australia etc. (1995).

[11] Meleshko V.V. Selected topics in the history of the two-dimensional biharmonic problem. Applied Mechanics Reviews. 56 (1), 33-85 (2003).

[12] Chekurin V.F. Variational method for solving direct and inverse problems of elasticity for a semi-infinite strip. Izv. Akad. Nauk. Mekh. Tverd. Tela. 2, 58-70 (1999). [Mech. Solids (Engl. Transl.) 34 (2), 49-59 (1999)].

[13] Chekurin V.F., Postolaki L. I. A variational method for the solution of biharmonic problems for a rectangular domain. Journal of Mathematical Sciences. 160 (3), 386-399 (2009).

[14] Kantorovich L. V., Krylov V. I. Approximate methods of higher analysis. Translated from the 3rd Russian Edition by C. D. Benster. New York, Interscience Publ. (1958).

[15] Chekurin V.F., Postolaki L. I. Theoretical-experimental determination of residual stresses in plane joints. Material Science. 45, 318-328 (2009).

[16] Chekurin V.F., Pokhmurs'ka H. V. Field of residual stresses in a coating with crack. Materials Science. 42 (2), 233-242 (2006).

[17] Chekurin V.F. Inverse problem of nondestructive control of the level of hardening of sheet glass. Mechanics of solids. 33 (3), 68-77 (1998).

[18] Chekurin V.F. Variational method for the solution of the problems of tomography of the stressed state of solids. Materials Science. 35 (5), 623-633 (1999).

[19] Chekurin V.F., Kravchyshyn O. Z. Inverse problem for acoustical tomography of stress fields in piecewisehomogeneous strip. In Proceedings of 8th International Seminar. Workshop on Direct and Inverse Problems of Electromagnetic and Acoustic Wave Theory DIPED (2003). 
[20] Chekurin V.F., Postolaki L.I. Variational method of homogeneous solutions in axisymmetric elasticity problems for a semiinfinite cylinder. Journal of Mathematical Sciences. 201 (2), 175-189 (2014).

[21] Chekurin V.F., Postolaki L. I. A variational method of homogeneous solutions for axisymmetric elasticity problems for cylinder. Mathematical modeling and Computing. 2 (2), 128-132 (2015).

[22] Chekurin V., Postolaki L. Application of the least square method in axisymmetric biharmonic problems. Mathematical Problems in Engineering. 2016, Article ID 3457649, 1-9 (2016).

[23] Chekurin V., PostolakiL. Residual stresses in a finite cylinder. Direct and inverse problems and their solving using the variational method of homogeneous solutions. Mathematical Modeling and Computing. 5 (2), 119-133 (2018).

[24] Saad H. M. Elasticity. Theory, Applications and Numerics. Academic Press (2005).

[25] Arfken G. "Hankel Functions." $§ 11.4$ in Mathematical Methods for Physicists, 3rd ed. Orlando, FL: Academic Press, pp. 604-610 (1985).

[26] Nowacki W. Theory of Elasticity. Moscow, Mir (1975), (in Russian).

[27] Aben H. Integrated Photoelasticity. New York, McGraw-Hill (1979).

[28] Chekurin V.F. Integral photoelasticity relations for inhomogeneously strained dielectrics. Mathematical Modeling and Computing. 1 (2), 144-155 (2014).

\title{
Осесиметрична задача теорії пружності для порожнистого циліндра $з$ ненавантаженими основами. Аналітичне розв'язування із використанням варіаційного методу однорідних розв'язків
}

\author{
Чекурін В. $\Phi .{ }^{1,2}$, Постолакі Л. I. ${ }^{1}$ \\ ${ }^{1}$ Інститут прикладних проблем механіки і математики ім. Я. С. Підстригача НАН Украӥни, \\ вул. Наукова, 3-б, Львів, 79060, Україна \\ ${ }^{2}$ Куявсъко-Поморсъкий Університет у Бидгощі, \\ вул. Торунъсъка, 55-5\%, Бидгощ, 85-023, Польща
}

\begin{abstract}
Розглянуто осесиметричну задачу для порожнистого циліндра із ненавантаженими основами. На внутрішній і зовнішній циліндричних поверхнях задано нормальні і тангенціальні навантаження. Задачу зведено до бігармонічного рівняння з відповідними крайовими умовами. За допомогою методу відокремлення змінних отримано однорідну крайову задачу для звичайного диференціального рівняння. Використовуючи власні функції цієї задачі, побудувано систему однорідних розв'язків вихідної бігармонічної задачі. Ї̈̈ розв'язок, який поданий як розвинення за цими функціями, залежить від чотирьох безмежних послідовностей невизначених дійсних констант. Для визначення невідомих констант застосовано варіаційний метод, згідно з яким підпорядкування розв'язку крайовим умовам, що задані на циліндричних поверхнях, здійснюється не поточково, а "в середньому" - за нормою $L_{2} .3$ цією метою введено функціонал, який визначає середньоквадратичне відхилення розв'язку від крайових умов, що задані на циліндричних поверхнях. У результаті отримано безмежну систему алгебраїчних рівнянь, яку розв'язано за допомогою методу редукції. Проведені кількісні дослідження підтвердили добру збіжність методу.
\end{abstract}

Ключові слова: задачі теорї пружності, порожнистий ииліндр, варіаційний метод однорідних розв'язків.

Mathematical Modeling and Computing, Vol. 7, No.1, pp. 48-63 (2020) 